\title{
Introduction to special issue on cardiac metabolism in hypertrophy and failure
}

\author{
William C. Stanley
}

Published online: 18 September 2013

(C) Springer Science+Business Media New York 2013

Interest has been growing over the last dozen years in understanding the role of myocardial energy metabolism in the development and progression of heart failure. Clinical efforts have focused on using indices of cardiac metabolic dysfunction to diagnose heart failure and optimize treatment strategies, and to develop pharmacological interventions to treat this malignant disorder. This important topic was addressed in a 2002 Special Issue of Heart Failure Reviews entitled "Myocardial Energy Metabolism in Heart Failure" [1]. Over the last decade, there has been much progress in our understanding of the role of energy metabolism in the pathophysiology of heart failure, and thus now, it is time for an update.

This special issue on "Cardiac Metabolism in Hypertrophy and Failure" presents recent advances in this area and aims to bring you up to date. These reviews emerged from the presentations given at the 10th Annual Meeting of the Society for Heart and Vascular Metabolism, which was held at Merton College at the University of Oxford. This outstanding conference was organized by Kieran Clarke, Stefan Neubauer, and colleagues, and provided attendees with an outstanding presentation of the latest advances in this important area of heart failure research. While there are many aspects of myocardial energy metabolism in hypertrophy and failure that remain poorly understood and are currently under intense investigation, there have also been meaningful advances in recent years. It has been long appreciated that cardiac metabolism is significantly perturbed in heart failure and that metabolic and bioenergetic modulation remains a potential therapeutic target. The papers presented in this special issue present the latest techniques for evaluating cardiac metabolism in humans, explore novel signaling mechanisms that regulate metabolism in the failing heart, and examine in depth the mitochondrial adaptations in response to hypertrophy and failure. In addition, the impact of heart failure on skeletal muscle metabolism is reviewed, as well as the latest finding on the effects of adipocyte-derived hormones on the heart.

Medical treatments for heart failure have changed little over the last decade and are still primarily aimed at symptom relief and with suppressing the over activation of neural-hormonal systems. These therapies only slow the progression of the disease, and thus, there is clearly room for novel drugs targeted to optimizing energy metabolism and preserving mitochondrial function in the failing heart. It is hoped that the papers in this Special Issue of Heart Failure Review will shed light on the critical questions in the field, and I hope it will inspire others to pursue new directions in this emerging area of heart failure research.

\section{Reference}

1. Stanley WC (2002) Introduction to special issue on myocardial energy metabolism in heart failure. Heart Fail Rev 7:113
W. C. Stanley $(\bowtie)$

Discipline of Physiology, University of Sydney, Anderson Stuart

Building (F13), Sydney, NSW 2006, Australia

e-mail: wstanley@usyd.edu.au 